\title{
Precise Truss Assembly using Commodity Parts and Low Precision Welding
}

\author{
Erik Komendera, Dustin Reishus \\ Department of Computer Science \\ University of Colorado at Boulder \\ Boulder, CO 80309
}

Email: firstname.lastname@colorado.edu

\author{
John T. Dorsey, William R. Doggett \\ NASA Langley Research Center \\ Hampton, VA 23681
}

Email: \{john.t.dorsey,bill.doggett $\} @$ nasa.gov

\author{
Nikolaus Correll \\ Department of Computer Science \\ University of Colorado at Boulder \\ Boulder, CO 80309
}

Email: nikolaus.correll@colorado.edu

\begin{abstract}
We describe an Intelligent Precision Jigging Robot (IPJR), which allows high precision assembly of commodity parts with low-precision bonding. We present preliminary experiments in $2 \mathrm{D}$ that are motivated by the problem of assembling a space telescope optical bench on orbit using inexpensive, stock hardware and low-precision welding. An IPJR is a robot that acts as the precise "jigging", holding parts of a local assembly site in place while an external low precision assembly agent cuts and welds members. The prototype presented in this paper allows an assembly agent (in this case, a human using only low precision tools), to assemble a $2 \mathrm{D}$ truss made of wooden dowels to a precision on the order of millimeters over a span on the order of meters. We report the challenges of designing the IPJR hardware and software, analyze the error in assembly, document the test results over several experiments including a large-scale ring structure, and describe future work to implement the IPJR in 3D and with micron precision.
\end{abstract}

\section{INTRODUCTION}

We present a hardware prototype of an Intelligent Precision Jigging Robot (IPJR) for assembling high-precision trusses from low precision components. IPJRs collaborate with external assembly agents by holding components precisely in place while they are welded by the external agent. This enables high precision and accuracy, despite using inexpensive stock components and low precision external manipulation.

High-precision trusses are a critical component in space telescopes, where they serve as optical benches, as well as many terrestrial applications ranging from buildings, bridges, and cranes. Space telescopes that could be assembled on orbit are a high priority for NASA [1]-[6], with proposed diameters ranging from $14 \mathrm{~m}$ (Figure 1) up to hundreds of meters; in contrast, the James Webb Space Telescope, at $6.5 \mathrm{~m}$, is the largest telescope that can be launched preassembled. Telescope mirrors usually consist of multiple mirror elements that are mounted onto a truss with parabolic curvature. The truss needs to be constructed such that the mounting points for the mirrors follow the desired parabolic curve as precisely as possible; how these mounting points are connected, however, is less important, motivating the approach presented in this paper. Ground-based robotic experiments [7] have demonstrated the repeated assembly and disassembly of an $8 \mathrm{~m}$ telescope mirror. While this approach has worked, it is considered impractical as it requires considerable launch mass, high precision on every member, and is inflexible to unexpected environmental effects. Other robot assembly methods have shown success

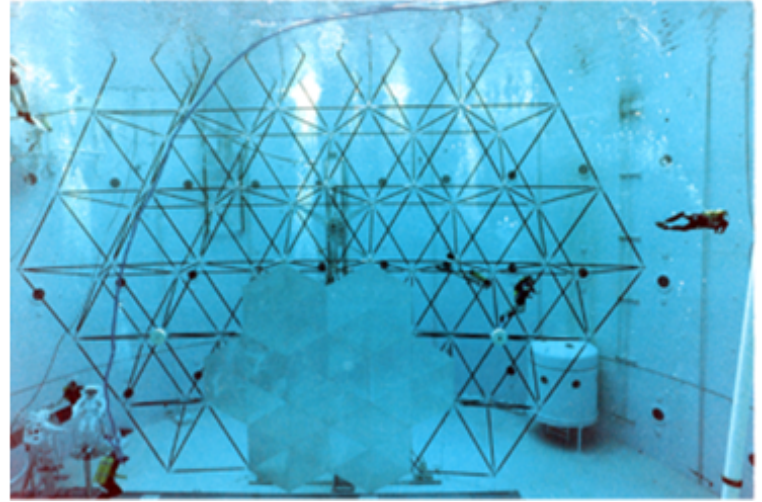

Fig. 1. A $14 \mathrm{~m}$ telescope mirror assembled in neutral buoyancy by EVA [6] using precision elements. Intelligent Precision Jigging Robots might allow to construct trusses with similar accuracy using commodity parts and lowprecision bonding techniques.

in robustness and cheapness of parts, but are limited to low precision and (mostly) homogeneous simple parts. Recent robust and parallel assembly techniques include quadrotor teams that can assemble cubic truss structures [8], truss climbing and assembling robots [9], termite-inspired swarm assembly robots [10], [11], and a robot team that can build IKEA furniture in cluttered environments [12]. While all of these robost approaches show promise, none currently cross the gap between concept and practicality.

The intelligent precision jigging paradigm is an attempt to close this gap. By incorporating the precision requirement into a welding jigging device, parts can be drawn from a stockpile of struts and nodes. External manipulators need not be precise, thereby separating the requirements between robots of different types and reducing the complexity of each type of robot. IPJRs can compensate for various problems in the assembly process, such as thermal expansion and, equipped with appropiate longrange sensors, error propagation. This system has the potential to solve both the problem of impracticality in previous robotic telescope assembly experiments, and the problem of precision assembly in the robust, parallel approaches.

This paper reports on the initial tests of a simple twodimensional IPJR prototype. These experiments validate the precision assembly of inexpensive materials. They also provide important lessons with respect to the design of more precise IPJRs for assembly of three-dimensional structures. Our prototype can assemble a truss made of wooden dowel rods to an 


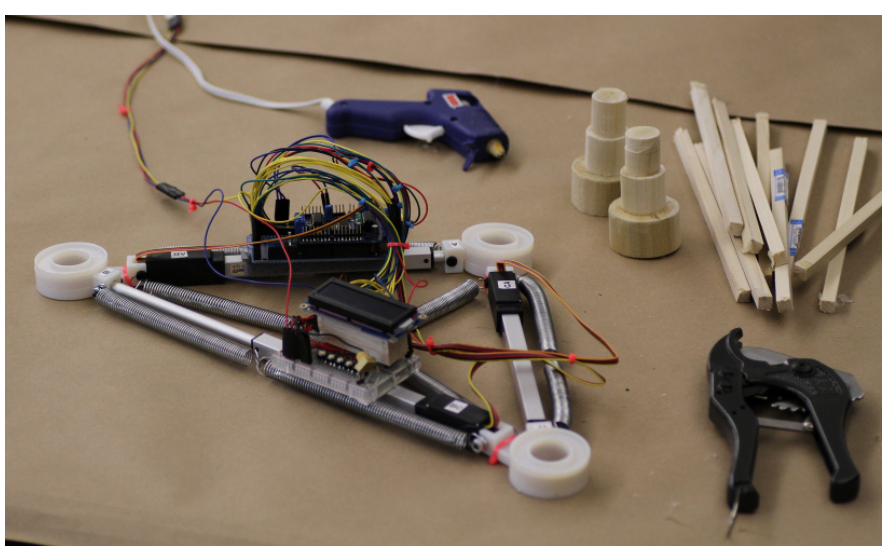

Fig. 2. The IPJR triangle in right triangle configuration. The controller is attached to the top-left edge, the button interface and LCD are attached to the bottom edge. The three joints are printed parts. Also in the picture (clockwise from top): the glue gun used to bond struts to nodes, nodes that form the vertices of the triangular cells, struts that form the edges of the triangular cells, and the low-precision cutting tool used to cut the struts to approximately the correct size. No other measurement, bonding, or cutting tools were used in the construction of the structure.

average accuracy of less than $1 \mathrm{~mm}$ over structures in which struts range from $290-430 \mathrm{~mm}$. The IPJR guides the assembly while forming the shapes of the constituent cells. A human assembly agent stands in for an external robot to cut and glue the pieces together. The human agent does not require other precision instruments or knowledge of what is being built; he visually estimates the strut lengths, fills the gaps with glue, and repositions the IPJR following commands on its display. This leads to a precise assembly that is accurate to the margins of error of the IPJR's sensors and actuators.

\section{InTELLIGENT PRECISION JigGING}

In welding and other applications, a jig is the name for a scaffolding device that ensures precision and repeatability. An "intelligent jig" is a jig with some amount of autonomy, capable of sensing, actuation, computation, and communication. These capabilities are intended to make the assembly task easier, faster, and more reliable. The simplest intelligent jig is one that can set and detect its own shape and alert the user when its requirements are met.

Trusses are usually made of triangular cells, often forming tetrahedra when embedded in three dimensions. Intelligent jigs for the assembly of these trusses should ensure that each node is located and aligned properly and that each strut, when welded, bears primarily an axial load between nodes. A jig for an individual strut should set the distance between the two nodes on either end to a desired precision and hold the strut steadily while the user welds the strut to both nodes. To form triangular and tetrahedral cells, it is sufficient that a group of connected intelligent jigs - using pin-joints in 2D and universal joints in 3D-set their edge lengths. The angles between struts will default to the unique solutions for triangles and tetrahedra of specified lengths. The user can reuse a triangular/tetrahedral group of intelligent jigs to assemble each cell independently, meaning that the complexity of the robot need not grow with the size of the structure.

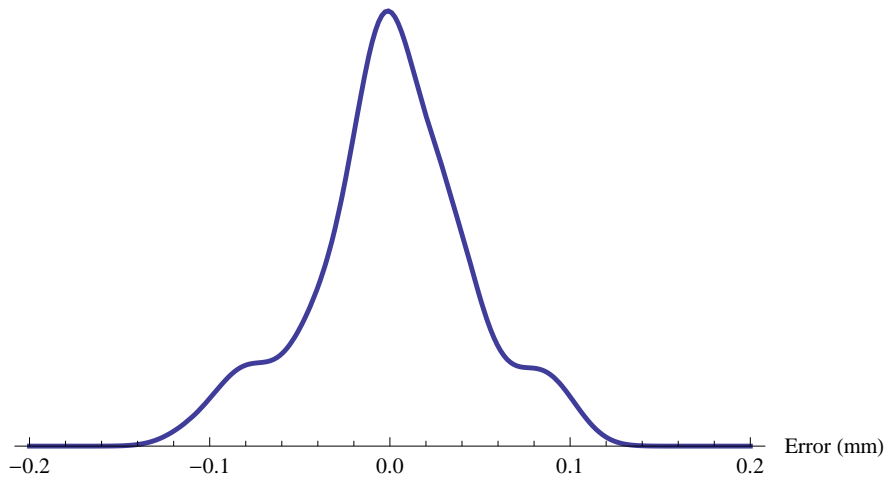

Fig. 3. Distribution on length errors in a single actuator.

The Intelligent Precision Jigging Robot is an implementation of truss jigging that assembles one cell at a time. Three jigs can connect to make a triangle, six a tetrahedron. They can communicate with each other and the external assembly agents, enabling them to discover the status of each other and coordinate the assembly process. In addition, several other tasks in the assembly process fall under the purview of the IPJRs including the sequencing assembly steps, determining and setting the lengths of each edge for each cell, and detecting when each step is complete (along with errors in each step). The external agent, i.e., a human user or a robot, only needs to know how to execute the orders given by the IPJRs.

\section{IPJR PROTOTYPE HARDWARE DESIGN}

The IPJR presented in this paper consists of a triangle of three actuators and is designed for precise assembly of wooden trusses. Figure 2 shows the IPJR prototype, assembly tools, struts, and nodes. Wooden dowels, acting as trusses, can be roughly cut and bound with glue by a human user. This system allows us to construct triangles with varying dimensions, and relies on an external agent to handle the struts and provide mobility to the jig.

The IPJR triangle's actuation consists of three Firgelli L16-140 150:1 -P actuators with built-in distance sensors in the form of potentiometers. This allows each jig to change its length from roughly $287 \mathrm{~mm}$ to $428 \mathrm{~mm}$. The control hardware is an Arduino Mega 2560 with an Adafruit motor shield, a $2 \times 16$ character LCD, and five buttons. Each actuator potentiometer has a stated accuracy of $0.5 \mathrm{~mm}$. Potentiometer readings are converted to a 10 bit signal (1024 discrete values) using the Arduino's analog-digital converter. Both actuator and joints have free play of about $1 \mathrm{~mm}$, which we address by attaching compressive springs to each edge and across the triangle. The joints are labeled $A, B, C$, and the edges are labeled $\overline{A B}, \overline{B C}, \overline{C A}$. Each joint has a $25.4 \mathrm{~mm}$ diameter cylindrical hole to align with a node on the structure.

We calibrated the distance potentiometers on each actuator by measuring the lengths for voltage levels spaced at intervals of 128 levels, along with levels 1 and 1022 to compensate for nonlinearity at the extremes. When calibrated, the minimum and maximum lengths of the edges, center of joint to center of joint of the triangular IPJR used in this paper, are respectively $287.0 \mathrm{~mm}$ and $428.0 \mathrm{~mm}$, giving the IPJR triangle a maximumto-minimum edge ratio of 1.49 , allowing the formation of 

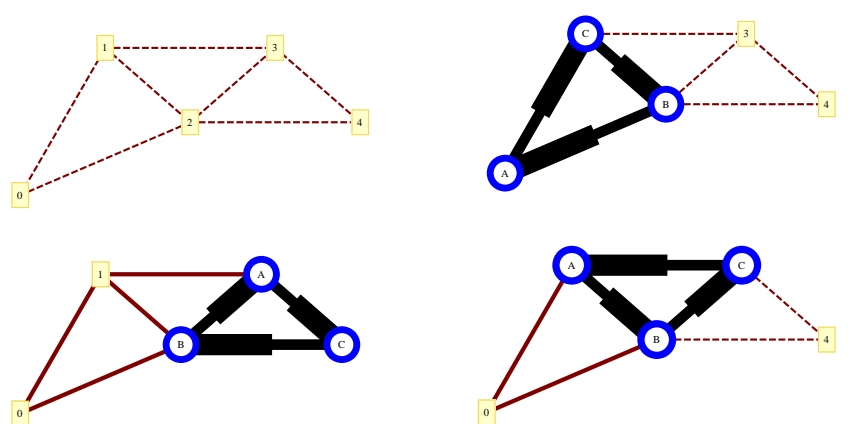

Fig. 4. A sample assembly sequence. Starting at top left with an assembly plan (dotted lines) and going clockwise, the IPJR triangle is placed at the site of the first intended triangle, the struts are bonded, the IPJR pivots clockwise around $\mathrm{B}$, the second cell is bonded, the IPJR pivots clockwise around B again, and the final cell is bonded.

$45^{\circ}-45^{\circ}-90^{\circ}$ right triangles. The springs reliably return the actuators to the expected voltage levels after the application and release of external forces on the IPJR triangle.

We used a Keyence IL-030 analog laser sensor and a Keyence IL-1000 amplifier unit to characterize the accuracy and repeatability of the actuators. The IL-030 has an accuracy of $1 \mu \mathrm{m}$. We set the actuators to a specific length $L$, tared the sensor, moved the actuator a random distance in $\pm 13 \mathrm{~mm}$, then back to $L$, and measured the offset using the IL-030. The distribution on the error, denoted $\mathcal{D}$, is shown in Figure 3, and has standard deviation $0.066 \mathrm{~mm}$.

\section{A. IPJR Forward Kinematics}

The IPJR's geometry is uniquely defined by the position of its three node centers $A=\left(x_{1}, y_{1}\right), B=\left(x_{2}, y_{2}\right)$ and $C=\left(x_{3}, y_{3}\right)$ in the plane, but has only three degrees of freedom, the length of the linear actuators $\overline{A B}=l_{1}, \overline{B C}=l_{2}$ and $\overline{C A}=l_{3}$. We therefore introduce a local coordinate system that has its origin at $\left(x_{1}, y_{1}\right)$ center and its $x$-axis parallel to one of the IPJR's legs. This approach leaves three free parameters, as three coordinates remain zero. For simplicity, we define the global coordinate system to be incident with the local coordinate system of the first triangle that the IPJR constructs.

We can now express the IPJR's local coordinates as

$$
\begin{aligned}
& x_{1}=0 \quad x_{2}=l_{1} \quad x_{3}=\frac{\frac{l_{1}^{2}+l_{3}^{2}-l_{2}^{2}}{2 l_{1}}}{y_{1}=0} \quad y_{2}=0 \quad y_{3}=\sqrt{l_{3}^{2}-\left(\frac{l_{1}^{2}+l_{3}^{2}-l_{2}^{2}}{2 l_{1}}\right)^{2}}
\end{aligned}
$$

The forward kinematics of the robot can also be used to calculate the expected error for each node in the structure using the error propagation law. Deriving analytical results is cumbersome, however, as error propagation depends on the order in which the structure is assembled. We therefore simulate the expected error for specific assembly sequences by drawing random edge lengths from the distribution $\mathcal{D}$, with mean at the desired distance.

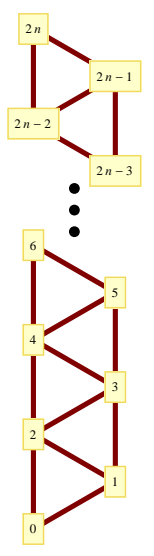

(a)

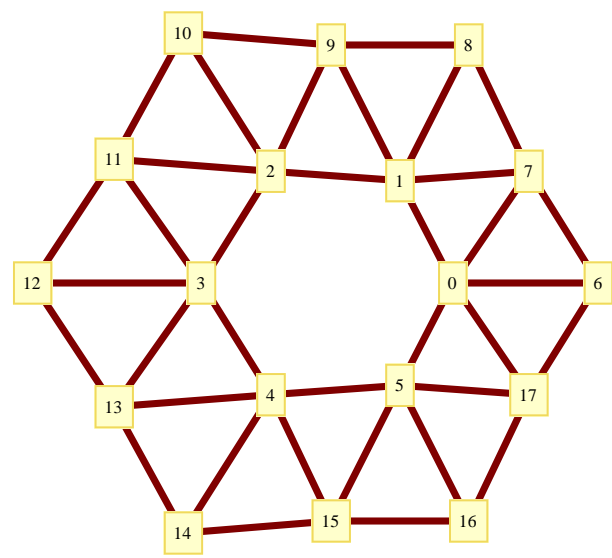

(b)
Fig. 5. Linear structure of length $n$ (a), and circular structure (b).

\section{Assembly Sequence And Control}

The IPJR triangle is used to assemble trusses one triangle at a time such that each new cell is adjacent to the previously built cell. Each triangle in a cell shares an edge and two nodes; this allows the transition command to be simple: "pivot IPJR triangle \{clockwise, counterclockwise\} around joint $\{A, B, C\}$ ". The user (or external robot) only needs to know how to follow that command. Figure 4 shows a complete assembly sequence for a simple structure.

The assembly sequence consists of a list of elements, one element per triangle that is assembled. For each triangle, the following information is needed: the lengths of each IPJR edge, and the transition pivot direction and joint from the previous cell. This information can easily be generated from an assembly blueprint, e.g., using depth-first search on the graph. For the first cell, the user places the IPJR triangle on three free nodes, and bonds the struts. The user lifts the IPJR triangle off the structure and the IPJR triangle modifies its edge lengths. Once the lengths are set, the user follows the transition command, positions the new nodes, and sets the IPJR triangle down onto the proper nodes. The user then bonds the new struts. This process continues until finished. For the initial prototype, the assembly sequence for our test structures was preprogrammed. Thus, for each assembly step, the order of substeps is: lift IPJR triangle, change lengths, pivot around a joint and set IPJR on the nodes, and bond struts.

The IPJR's actuators use a modified bang-bang controller to drive the joints to the desired length, as measured by the potentiomenter voltage level. Typically, it requires less than 2 seconds to converge after the initial approach, and never failed to converge in any of our experiments. While this simple controller is sufficent for the experiments presented here, convergence time can be improved possibly further by designing an optimal controller, which is not the scope of this paper, however.

\section{EXPERIMENT AND RESULTS}

We present three sets of experiments: simulation results that illustrate error propagation for two extreme cases, a linear 


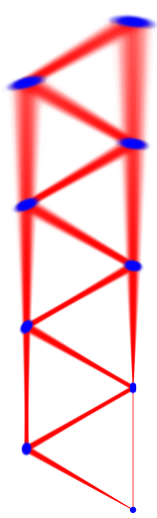

(a)

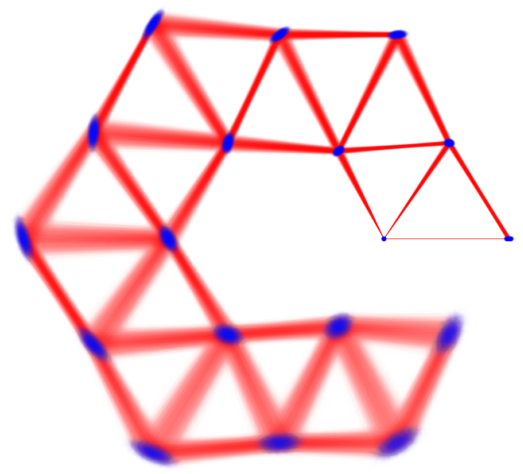

(b)
Fig. 6. Simulation of error propagation for a linear truss (a) and a circular truss (b). Shades correspond to the likelihood of a specific configuration (dark most likely). Errors have been amplified by a factor of ten for illustration purposes.

truss and a ring; repeated assembly of a simple, four-triangle square to study accuracy experimentally; and the assembly of a single large ring-like truss. Future work will explore external positioning and error correction systems; in the experiments presented in this paper, accuracy is driven only by the IPJR.

\section{A. Simulation results}

To get a better understanding of how errors propagate during assembly, we simulated the construction of different truss structures, modeling the edge length and error distribution with values experimentally obtained from the IPJR, as shown in Figure 3. The simulation used the coordinate system as defined in Section III-A. We simulated adding one triangle at a time with edge lengths randomly drawn from the distribution, $\mathcal{D}$, plus the desired length.

We first simulated a linear truss structure, as shown in Figure 5 (a). The ideal edge lengths were $350 \mathrm{~mm}$ to which random noise was added. The results of the simulation were distributions of structures, whose average was the ideal structure. For $n=4$, the resulting structure distribution is shown in Figure 6 (a). The figure shows the bottom node in a fixed position, whereas the other nodes are distributed randomly, with darker shades indicating more likely positions. Note that the errors have been amplified by a factor of 10 in order for the distribution to be visible. The simulation was repeated for $n \in\{2, \ldots, 100\}$ and the average error of node $2 n$ was measured. The results are summarized in Figure 7. For small structures (less than $2 \mathrm{~m}$ long, or $n \leq 3$ ), the average absolute error was less than $0.2 \mathrm{~mm}$, giving an average relative error of less than $0.01 \%$. For larger structures (between $50 \mathrm{~m}$ and $60 \mathrm{~m}$, or $140 \leq n \leq 170$ ), the average absolute error was less than $3 \mathrm{~cm}$, giving an average relative error of less than $0.05 \%$.

In order to show that it would be possible to close a ring structure, we simulated assembling the structure shown in Figure 5 (b). If the accumulated error in the position of the final node led to it being farther from the initial node than the maximum extent of the actuators on the IPJR, then the IPJR could not act as a jig to close the ring. Figure 6 (b) shows how the error could accumulate to bring the final node too far from the initial nodes. Note that in order for the error distribution

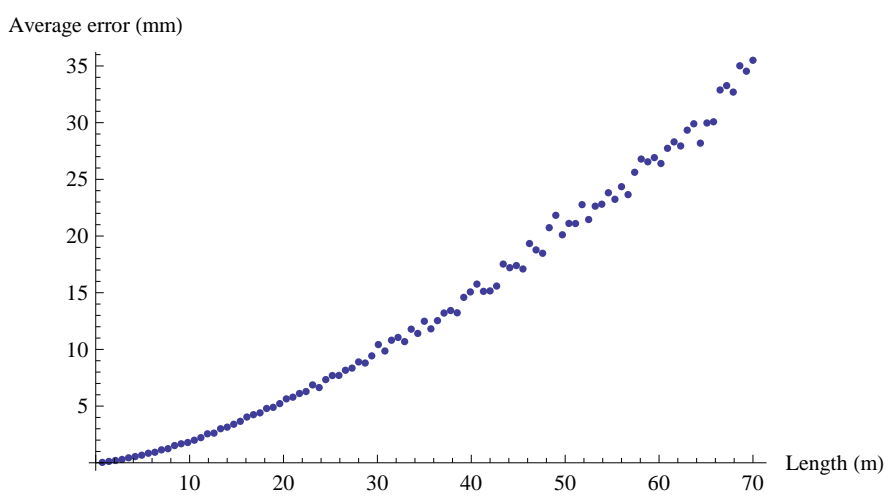

Fig. 7. Average error in the position of the last node in a linear structure $x$ meters long.

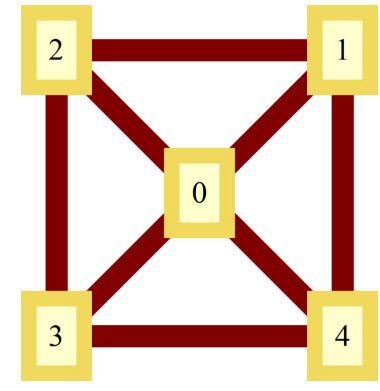

(a)

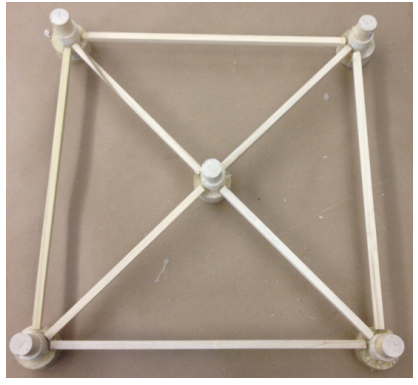

(b)
Fig. 8. Schematic of a square truss (a) and image of a completed square truss (b).

to be visible in Figure 6, we had to exaggerate it by a factor of ten. Using the measured error distribution on the actuators, we found the standard deviation of the length between the last node and the first was $0.4 \mathrm{~mm}$, well within the range of the IPJR.

\section{B. Wooden trusses: square and ring}

We cut struts from $12.7 \mathrm{~mm}$ square dowels. We construct nodes from $25.4 \mathrm{~mm}$ height segments of $50.8 \mathrm{~mm}, 31.75$ $\mathrm{mm}$, and $25.4 \mathrm{~mm}$ diameter cylinders respectively, forming a $76.2 \mathrm{~mm}$ step cone. Struts attach to the bottom step, the IPJR triangle rests on the second step, and distance measurements are based on the center of the top step. The topmost $25.4 \mathrm{~mm}$ cylinders fit in the ring-like joints on the IPJR triangle. When connecting struts to nodes, the struts are cut to length and are glued to the tops of the bottom cylinder.

1) Square: The square truss experiment was designed to test the reliable assembly of a large square subdivided into four right triangles. The design has five nodes: one at the center, and four at the corners. There are eight struts: four on the outside and four connecting the corners to the middle, as shown in Figure 8. The interior node-to-node distances should be 295 $\mathrm{mm}$, and the exterior node-to-node distances should be 417.2 $\mathrm{mm}$.

The four-step assembly sequence was as follows: the first cell requires joint $A$ on node $0, B$ on 1 , and $C$ on 2 . Then the cell rotates counterclockwise around joint $A$ three times. The final cell only requires the placement of the final outer strut. 


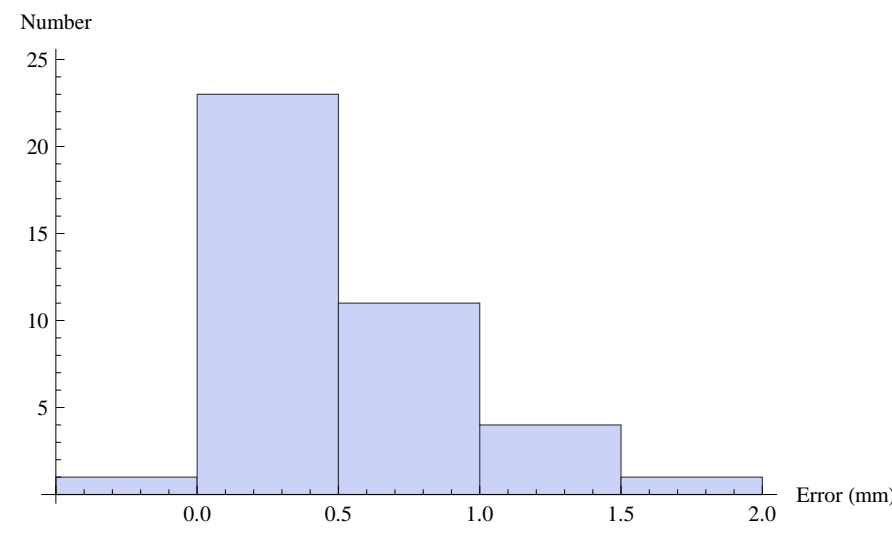

Fig. 9. Histogram of the measured edge length errors for five square truss assembly tests, in which the desired lengths are $295 \mathrm{~mm}$ and $417.2 \mathrm{~mm}$. The mean error is $0.416 \mathrm{~mm}$

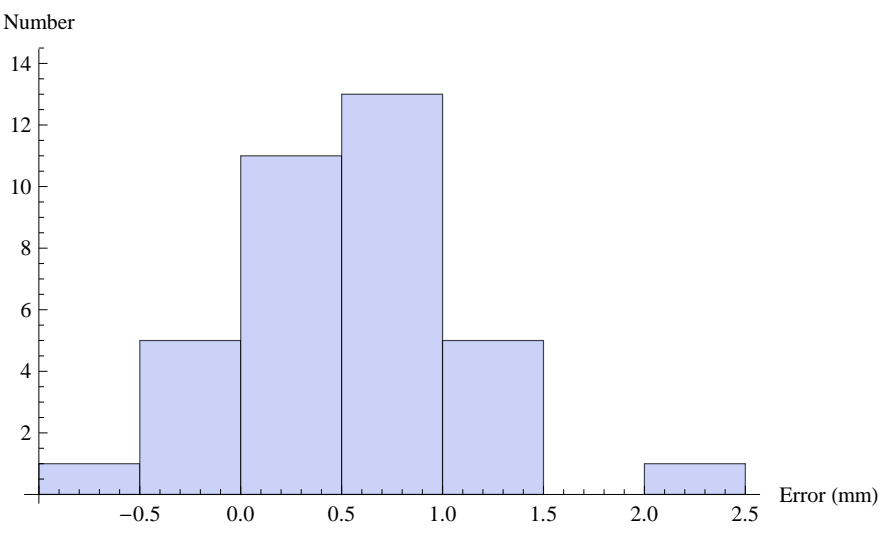

Fig. 10. Histogram of measured length errors of the physical experiment of the irregular ring truss. The mean error is $0.508 \mathrm{~mm}$.

This experiment was performed five times, and the distances between each node center were measured to a precision of 0.5 $\mathrm{mm}$.

The measured edge length errors, in Figure 9, show that the edges are longer than the nominal length. The average error is $0.416 \mathrm{~mm}$, with a standard deviation of $0.459 \mathrm{~mm}$. Strut $(4,1)$ was the final edge added, and it was the only edge that had no free nodes.

2) Ring Truss: Space telescope optical benches will not be composed of equilateral triangles; the geometry will be mapped to fit the surface of a parabola. The irregular ring truss test reflects this mapping and demonstrates the versatility of the robot. The truss, shown in Figure 5 (b), consists of equilateral triangles that are deformed such that the shortest struts, $(0,1)$ and $(0,5)$, are near the minimum IPJR edge length, and the longest strut, $(3,12)$, is near the maximum IPJR edge length. The structure is symmetrical around the axis defined by nodes $0,3,6$, and 12 . The assembly sequence started at triangle $(0,6,7)$ and proceeded around the ring counterclockwise, ending with triangle $(0,17,6)$. The pivot for every transition was a joint on the inner ring; if there were two, the one farther along the inner ring in the counterclockwise direction was chosen.

The average edge length error was $0.508 \mathrm{~mm}$, as shown in

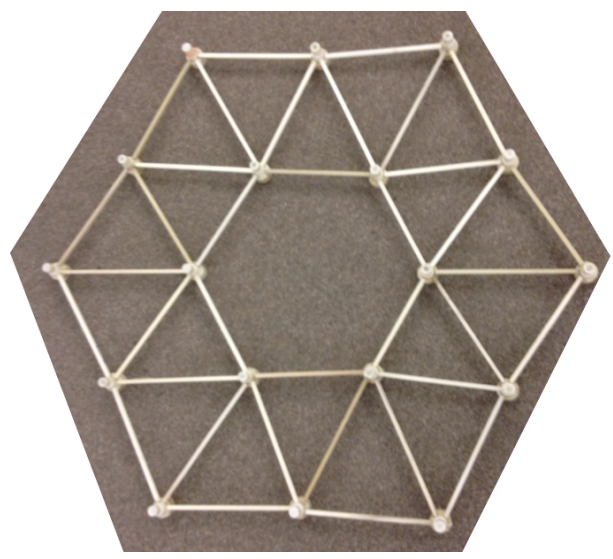

Fig. 11. Result of the ring truss assembly experiment.

Figure 10, and the standard deviation was $0.601 \mathrm{~mm}$. Prior to adding the final two triangles, the three unplaced edges $(0,5)$, $(0,17)$, and $(6,17)$ were measured, to calculate the overall drift of the structure. The gap errors came out to be $0.5 \mathrm{~mm}, 0.8$ $\mathrm{mm}$, and $1.4 \mathrm{~mm}$. The largest of these is within the range of other edge errors, and we did not need to apply stress on the structure to reduce the gap. Figure 11 shows the completed structure.

\section{DISCUSSION}

All $(4,1)$ struts in the square truss were at least $1 \mathrm{~mm}$ longer than expected. The positive length bias shown in both Figures 9 and 10 was a likely contributor: for any triangle, if a constant is added to all three lengths, the resultant angles will slightly bias toward $60^{\circ}$. A positive length bias would result in the first three right angles to be slightly less than 90 degrees, and the final angle having to be larger to close the structure, causing the increase.

On the ring truss, the wide range of errors on the other edges indicate its large errors canceled out in the accumulation. The one major anomaly was strut $(4,13)$, which was off by about $2.3 \mathrm{~mm}$. The assembly of the two triangles containing that strut proceeded like every other, and we noticed no obvious difference. Its symmetric strut, $(2,10)$, was off by $-0.5 \mathrm{~mm}$.

Other errors in the calibration could have also contributed. IPJR edge $\overline{B C}$ was the only edge used for the outer struts on the square truss, possibly leading to an increased bias versus the case where the IPJR rotated around the outer vertices. The calibration may have been altered by the springs exerting different forces for the right triangle, causing the error. The low precision strut measurement device may have contributed. We may have erred in calculating node centers, which were the basis for determining strut length. Finally, the small number of tests and the free play between nodes and struts could mean that these results are not indicative of the long term behavior, but both the square truss and the ring truss demonstrate the positive error bias. The size of the ring truss meant that it had to span multiple tables, whose surfaces were offset by a few millimeters.

While the accumulated error when constructing the ring did not exceed $1.4 \mathrm{~mm}$ for the final edges, we believe that 
by closing the loop, we can reduce the error throughout the structure. The five square truss tests and the one ring truss test were completed without having to induce stress on the trusses to close the final cells. These tests gave us valuable insights on how to improve the IPJR. The precision and accuracy could be improved by choosing a self-centering connection mechanism to connect the IPJR with the nodes instead of the node-withinloop configuration. We also observe that the potentiometer calibrations are not independent in practice. Length changes on one IPJR edge might induce subtle stresses that slightly change the readings without noticeably changing the actual edge length, leading to different offsets on the potentiometer output as a function of the length of the other actuators. The largest such error was $0.3 \mathrm{~mm}$, and is likely one of the largest sources of error in the experiments. This source of error was not modeled in the simulations. This error might be countered by performing a multi-variate calibration that takes the overall state of the system into account.

Moving to 3D requires us to overcome a series of mechanical challenges: first, each joint on the IPJR must be a universal joint with three attachment points and an additional three edges, i.e., for constructing tetrahedral structures. Second, we require a node design that can be precisely positioned by the IPJR, yet not interfere with the IPJR actuation. Third, unlike in 2D where the IPJR is congruent to the cell it is constructing, the choice must be made on whether the IPJRs should be inside the cell they are building, or whether there can be a mixture of inner and outer joints.

All presented structures can only be assembled in one way when symmetry is considered. However, trusses such as space telescope optical benches may have multiple build sequences, resulting in a wide range of maximum errors. Finding a method to minimize this error is key to utilizing the IPJR paradigm. Likewise, a method to detect and correct for errors during the assembly sequence can further improve the outcome, such as using an external, global error detection system.

\section{CONCLUSION AND FUTURE WORK}

We presented a prototype of an Intelligent Precision Jigging Robot (IPJR), which assists users in constructing precise triangular trusses using low-cost and low-precision assembly methods. Final truss assembled precision is driven by the accuracy of the IPJR's sensors and actuators and is dependent on the actual truss configuration.

The prototype was able to demonstrate the accurate and precise construction of different trusses by a user that followed commands issued by the IPJR. Nodes were constructed of cylindrical dowels, and struts were cut on the spot from lengths of rectangular dowels. The user cut and bound the struts to the nodes using only low precision and inexpensive tools. The IPJR served as a reliable and steady jig during the bonding phases. The accumulated error over each test was small enough that the loop closure was possible without having to load and deform the structure to place the final struts. The strut errors had a $0.5 \mathrm{~mm}$ bias toward being longer, but some edges were off by more than a millimeter, while only one was off by more than $2 \mathrm{~mm}$. We identified several sources of error, and intend to correct them.
Future work will be geared toward building a full-scale, 3D telescope optical bench. We will extend the IPJR concept to higher precision using appropriate sensors, actuators, and connection mechanisms, and will replace the human operator by a remote controlled robot arm [13], making the system fully autonomous. The wooden parts will be replaced by node balls having a precise reference point (the mirror attachment point), and the struts will be replaced by telescoping tubes that can be welded shut when finalized. We will also explore methods to both plan assembly sequences that minimize error, and correct error accumulation during the assembly. Finally, we wish to explore parallel algorithms enabling swarms of IPJRs and external agents to build large-scale structures on orbit both efficiently and robustly.

\section{ACKNOWLEDGMENTS}

This work was supported by a NASA Office of the Chief Technologist's Space Technology Research Fellowship.

\section{REFERENCES}

[1] W. R. Oegerle, L. R. Purves, J. G. Budinoff, R. V. Moe, T. M. Carnahan, D. C. Evans, and C. K. Kim. Concept for a large scalable space telescope: In-space assembly. Proceedings of SPIE, 6265(62652C), 2006.

[2] D. Ebbets, J. DeCino, and J. Green. Architecture concept for a 10m uvoptical space telescope. Proceedings of SPIE, 6265(62651S-1), 2006.

[3] M. S. Lake. Launching a 25-meter space telescope, are astronauts a key to the next technically logical step after ngst? In IEEE Aerospace Conference, 2001.

[4] M. J. Mahoney and A. C. Ibbott. A large deployable reflector assembly scenario, a space station utilization study. Technical Report NASA JPL D-5942, 1988

[5] C. F. Lillie. On-orbit assembly and servicing of future space observatories. Proceedings of SPIE, 6265(6265 62652D-1), 2006.

[6] J. J. Watson, T. J. Collins, and H. G Bush. Construction of large space structures at NASA Langley Research Center. In IEEE Aerospace Conference, 2002.

[7] W. Doggett. Robotic assembly of truss structures for space systems and future research plans. In Aerospace Conference Proceedings, 2002. IEEE.

[8] Q. Lindsey, D. Mellinger, and V. Kumar. Construction of cubic structures with quadrotor teams. In Proceedings of Robotics: Science and Systems, June 2011.

[9] C. Detweiler, M. Vona, Y. Yoon, S. Yun, and D. Rus. Self-assembling mobile linkages. Robotics \& Automation Magazine, IEEE, 14(4), 2007.

[10] J. Werfel and R. Nagpal. Three-dimensional construction with mobile robots and modular blocks. International journal of robotics research, 3-4(27), 2008.

[11] K. Petersen, R. Nagpal, and J. Werfel. Termes: An autonomous robotic system for three-dimensional collective construction. In Robotics: Science and Systems VII, 2011.

[12] R.A. Knepper and D. Rus. Pedestrian-inspired sampling-based multirobot collision avoidance. In Proceedings of the International Symposium on Robot and Human Interactive Communication (RO-MAN), 2012.

[13] J. T. Dorsey, T. C. Jones, W. R. Doggett, J. S. Brady, F. C. Berry, G. G. Ganoe, E. J. Anderson, B. D. King, and C. D. Mercer. Recent developments in the design, capabilities and autonomous operations of a lightweight surface manipulation system and test bed. In Proceedings of the AIAA Space Conference, 2011. 